\title{
A Novel Feature Detection Algorithm Based on Improved 2DPCA-SIFT
}

\author{
AiliWang ${ }^{1}$, Yangyang Zhao ${ }^{1}$, Jiaying Zhao ${ }^{1}$, Yuji Iwahori ${ }^{2}$ and Xinyuan Wang ${ }^{1}$ \\ ${ }^{1}$ Higher Education Key Lab for Measuring \& Control Technology and \\ Instrumentations of Heilongjiang, Harbin, China \\ ${ }^{2}$ Dept. of Computer Science, Chubu University, Japan \\ aili925@hrbust.edu.cn
}

\begin{abstract}
Stable local feature and representation is a fundamental component of many image registration, $3 D$ reconstruction and object recognition algorithms. SIFT is a good descriptor that encodes the salient aspects of the image gradient in the feature point's neighborhood. This paper improved SIFT- based local image descriptor and proposed a SIFT feature matching algorithm based on improved 2DPCA which can eliminate both rows and columns of relevance. Experimental results show that improved 2DPCA-SIFT algorithm is relatively stable, accurate and fast.
\end{abstract}

Keywords: three-dimensional reconstruction, two-dimensional principal component analysis, SIFT feature matching

\section{Introduction}

In the field of three-dimensional reconstruction, each image contains a lot of information based on the presence of image pixels, but not all of the pixel can produce three-dimensional reconstruction assistance. Only those particular points which are clearly different between the image points are referred to as feature points (feature point). In two images, get these two-dimensional coordinates of feature points, three-dimensional reconstruction can be obtained. Detection and extraction of feature points require automatic detection technology. Feature point detection and matching process is inevitably error and mismatch, therefore, a robust strategy is needed to remove the mismatch effect, so that the reconstruction process is robust [1].

This paper presents a feature extraction algorithm based on improved two dimensions principal component-invariant feature transform (2DPCA-SIFT) for improved 2DPCASIFT algorithm inherits the traditional SIFT algorithm of stability and a large number of advantages, and two-dimensional principal component analysis reduced the traditional SIFT feature descriptor vectors of dimension and improves efficiency.

\section{Traditional SIFT Feature Matching Algorithm}

Traditional SIFT algorithm detects and find a local extremum in two-dimensional space and scale-space and extracts the key points for precise positioning. Then, according to the direction of the gradient direction of the neighborhood around the point to calculate the key direction to achieve operator and rotational invariance to geometric transformation [2]. General build SIFT feature descriptor takes a few steps: 1) scale-space establishment; 2) calculation of critical point features, feature point location; 3) the SIFT feature descriptor vectors expression [3]. 


\subsection{Establishment of Scale-Space}

Koenderink proved that scale transformation of Gaussian convolution kernel is a unique linear transformation kernel. Two-dimensional Gaussian function is defined as:

$$
\mathrm{G}(\mathrm{x}, \mathrm{y}, \mathrm{s})=\frac{1}{2 p s^{2}} e^{-\left(x^{2}+y^{2}\right)} / 2 s^{2}
$$

Where (x, y) is the space coordinates. Then, a two-dimensional scale space image can be obtained by convolution of image and Gaussian kernel function.

$$
\mathrm{L}\left(\mathrm{x}, \mathrm{y}, s_{D}\right)=\mathrm{G}\left(\mathrm{x}, \mathrm{y}, s_{D}\right) * \mathrm{I}(\mathrm{x}, \mathrm{y})
$$

Detection of characteristic points are processed in scale-space and the image space at the same time, in order to get robust feature points. DOG operator is introduced, which is two-scale difference value of the Gaussian kernel. DOG operator is shown as following:

$$
D(x, y, s)=(G(x, y, k s)-G(x, y, s)) * I(x, y)=L(x, y, k s)-L(x, y, s)
$$

In this stage, the locations of potential interest points in the image are determined by detecting the extrema of Difference of Gaussian (DoG) scale space. For searching scale space extrema, each pixel in the DoG images is compared with its 26 neighbors in $3 \times 3$ regions of scale-space. If the pixel is lower/larger than all its neighbors, then it is labelledas a candidate key-point. Each of these key-points is exactly localized by fitting 3D quadratic function computed using a second order Taylor expansion around key-point location. Hence key-points are filtered by discarding points of low contrast and points that correspond to edges

\subsection{Computation the Feature Direction of Key Points}

In order to enable the extraction of feature with zoom and rotational invariance, it is necessary to use neighborhood around each point to calculate feature direction of the key point. Gradient value of key point is defined as:

$$
\mathrm{m}(\mathrm{x}, \mathrm{y})=\sqrt{(L(x+1, y)-L(x-1, y))^{2}+(L(x, y+1)-L(x, y-1))^{2}}
$$

The main direction of the key point is defined as:

$$
\theta(\mathrm{x}, \mathrm{y})=\partial \tan 2 \frac{L(x, y+1)-L(x, y-1)}{(x+1, y)-L(x-1, y)}
$$

\subsection{SIFT Feature Descriptor Vectors}

As shown in Figure 1, each feature point is regarded as the center and chooses a region with size of 16x16 pixels. Then depart the region into 4 blocks with size of $4 \times 4$ and calculate gradient histogram which contains has 8 bin directions to get 128 dimension vector, called SIFT features description. Histogram's peak is the principal directions of the selected feature point.
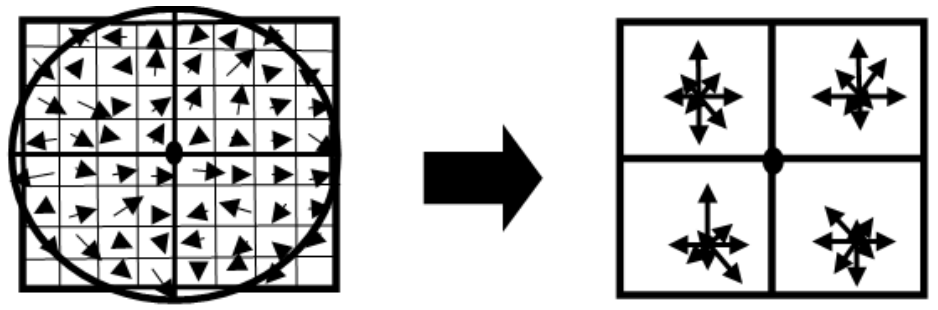

Figure 1. Sift Feature Descriptor Vectors 


\subsection{Improved 2DPCA-SIFT Feature Matching Algorithm}

Improved 2DPCA-SIFT algorithm [7] includes the basic processes: SIFT is used to extract key points, determine the location and size; Dimensionality reduction is used improved 2DPCA and construct feature descriptor based on characterization of improved 2DPCA[7]; Select the appropriate measure to calculate similarity and finally eliminate mismatch using RANSAC algorithm to get match results for image pair.

PCA removes the correlation between models and achieves data compression. However, PCA needs to decompose a non-sparse matrix; causing vector dimension of the image is too high and resulting in dimensionality disaster. Thus dimension reduction destroys the data of the original space topology, and PCA is unable to reveal nonlinear structures in the data structure [8]. Improved 2DPCA can solve these problems and the basic theory is introduced in the following paragraph.

\subsubsection{Theory of Improved 2DPCA}

The image matrix A size of $m \times n, X \in R^{n \times d}(n \geq d)$ is a matrix which column vectors are mutually orthogonal, linear transformation $\mathrm{Y}=\mathrm{AX}$, where $\mathrm{A}$ is projected onto the image matrix $X$, the projection $Y$ will have a feature vector of $m \times d$. With total dispersion of the sample as a criterion function projection $\mathrm{J}(\mathrm{X})$ can find the optimal projection matrixX:

$$
\mathrm{J}(\mathrm{X})=\operatorname{tr}\left(\mathrm{S}_{\mathrm{x}}\right)
$$

where $S_{x}$ is the covariance matrix projection matrix $Y, \operatorname{tr}\left(S_{x}\right)$ is a trace of $S_{x}$.

$$
\begin{aligned}
J(X)=\operatorname{tr}\left\{E\left[(A X-E(A X))(A X-E(A X))^{T}\right\}\right. & \\
= & \operatorname{tr}\left\{X^{T} E\left[(A-E A)^{T}(A-E A)\right] X\right\}
\end{aligned}
$$

Suppose the number of training samples is $M$, which is represented as $m \times n$ matrix $A_{l}$ $1=1,2 \ldots, M)$ and the mean of image is:

$$
\overline{\mathrm{A}}=\frac{1}{\mathrm{M}} \sum_{\mathrm{l}=1}^{\mathrm{M}} \mathrm{A}_{\mathrm{l}}
$$

The $\mathrm{G}$ is estimated as follows:

$$
\mathrm{G}=\frac{1}{\mathrm{M}} \sum_{\mathrm{l}=1}^{\mathrm{M}}\left(\mathrm{A}_{\mathrm{l}}-\overline{\mathrm{A}}\right)^{\mathrm{T}}\left(\mathrm{A}_{\mathrm{l}}-\overline{\mathrm{A}}\right)
$$

The largest eigenvalue of the matrix $G$ corresponding to the feature vector $X_{1}, X_{2}$, $\ldots X_{d}$ constituting the projection matrix $X_{\text {opt }} X$ is $X_{\text {opt }}=\left[X_{1}, \ldots X_{d}\right]$ is the optimal value. In the obtained projection matrix $\mathrm{X}_{\mathrm{opt}}=\left[\mathrm{X}_{1}, \ldots \mathrm{X}_{\mathrm{d}}\right]$ after the image can feature extraction and classification, and for a given image $\mathrm{A}$.

$$
\mathrm{Y}_{\mathrm{l}}=\mathrm{AX}_{1} \quad(\mathrm{l}=1,2, \quad \ldots \mathrm{d})
$$

To obtain a set of feature vectors after projection $L=\left[Y_{1}, \quad Y_{2}, \ldots Y_{d}\right]$ it is called the principal component vector image $A$. In fact, for (3) $A_{1}$ and $\bar{A}$, they can be written as follow

$$
\begin{gathered}
A_{l}=\left[\left(A_{l}{ }^{(1)}\right)^{T}\left(A_{l}^{(2)}\right)^{T} \ldots\left(A_{l}^{(m)}\right)^{T}\right]^{T} \\
\bar{A}=\left[\left(\bar{A}^{(1)}\right)^{T}\left(\bar{A}^{(2)}\right)^{T} \ldots\left(\bar{A}^{(m)}\right)^{T}\right]^{T}
\end{gathered}
$$

At this time $A_{l}{ }^{(j)}, \bar{A}^{(j)}$ respectively, the first vector $A_{-} 1 \mathrm{i}$ and $\mathrm{A}^{-}$trekking, so 
Now reorder:

$$
G=\frac{1}{M} \sum_{1 \leq 1 \leq M}\left(A_{l}^{(i)}-\bar{A}^{(i)}\right)^{T}\left(A_{l}^{(i)}-\bar{A}^{(i)}\right)
$$

$$
\begin{gathered}
A_{1}=\left[\left(A_{1}^{(1)}\right)\left(A_{1}^{(2)}\right) \ldots\left(A_{1}^{(m)}\right)\right] \\
\bar{A}=\left[\left(\bar{A}^{(1)}\right)\left(\bar{A}^{(2)}\right) \ldots\left(\bar{A}^{(m)}\right)\right]
\end{gathered}
$$

Here $\mathrm{A}_{\mathrm{l}}{ }^{\mathrm{j})}, \overline{\mathrm{A}}^{(\mathrm{j})}$ are the jth column and the column vector $\mathrm{A}_{\mathrm{l}}$ and $\overline{\mathrm{A}}$ so the covariance matrix $G$ based on the image in the column direction can be defined as

$$
G=\frac{1}{M} \sum_{1 \leq l \leq M}\left(A_{l}^{(j)}-\bar{A}^{(j)}\right)\left(A_{l}^{(j)}-\bar{A}^{(j)}\right)^{T}
$$

Get the eigenvalue $\lambda_{1}, \lambda_{2}, \ldots, \lambda_{n}$ of matrix and corresponding feature vector $v_{1}, v_{2}, \ldots, v_{n}$, sort and select the $\mathrm{r}$-th largest eigenvalue and feature vectors to form projection matrix as follow:

$$
\left\{\begin{array}{c}
w=\left(v_{1}, v_{2}, \ldots, v_{r}\right) \\
v_{i}^{T}=\left(v_{i 1}, v_{i 2}, \ldots, v_{i n}\right) \\
v_{i}^{T} v_{i}=1,(i=1,2, \ldots, n)
\end{array}\right.
$$

Through the linear transformation, image multiplies the projection matrix to generate improved 2DPCA-SIFT descriptor, thus make up a matrix size of $m \times r(39 \times 8)$ the $y$, called feature matrix of image A, as following:

$$
\left\{\begin{array}{l}
Y_{i A}=A_{i} w(i=1,2, \ldots, M)=\left[Y_{1}^{(i)}, Y_{2}^{(i)}, \ldots, Y_{r}^{(i)}\right] \\
Y_{j b}=B_{j} w(j=1,2, \ldots, N)=\left[Y_{1}^{(j)}, Y_{2}^{(j)}, \ldots, Y_{r}^{(j)}\right]
\end{array}\right.
$$

\subsubsection{Matching of Corresponding Feature Points Matching}

When two images using improved 2DPCA-SIFT feature extraction algorithms identify the individual characteristics and for precise positioning, after you generate the corresponding feature vector, you can calculate the eigenvectors of Euclidean distance between two smaller values of between eigenvectors of Euclidean distance, it means two more similar, their matching degree higher. Calculation of Euclidean distance formula is shown as:

$$
\mathrm{D}=\sqrt{\left(x_{1}-x_{1}^{\prime}\right)^{2}+\left(x_{2}-x_{2}^{\prime}\right)^{2}+\cdots+\left(x_{128}-x_{128}^{\prime}\right)^{2}}
$$

Where $\left(x_{1}, x_{2}, \ldots \ldots, x_{128}\right),\left(x_{1}^{\prime}, x_{2}^{\prime}, \ldots \ldots, x_{128}^{\prime}\right)$ respectively are feature vectors of key points. First find a point in one of the images, use traversal method to find the nearest point and sub-nearest point in another image. If the two points satisfy the relationship in the following inequality, they are regarded as matching. The inequality is shown as:

$$
\frac{D_{\text {nearest }}}{D_{\text {hpyo-nearest }}}<r
$$

where $D_{\text {nearest }}$ and $D_{\text {hpyo-nearest }}$ are the nearest and the sub-nearest Euclidean distance respectively, and the threshold $\mathrm{r}$ is chosen as 0.6 in experiments.

\section{Experimental Results and Analysis}

Experimental environment is CPU Intel $\mathrm{I} 52.6 \mathrm{GHz}, 4 \mathrm{Gbyte}$ memory, video memory is 2Gbyte, the operating system for Windows 8.1, simulation platform is Matlab2012B. Images with different background are used in the experiment. Improved 2DPCA-SIFT proposed in this paper and PCA-SIFT algorithm are compared to evaluate matching 
validity and computation time. Matching results of image toy by different algorithm are shown in Figure 2 and Figure 3 separately, and those of image telecontroller are shown in Figure 4 and Figure 5. Red points mean matching errors. It can be seen, PCA-SIFT produced more obviously errors, and improved 2DPCA-SIFT algorithm reduced probability of matching errors.

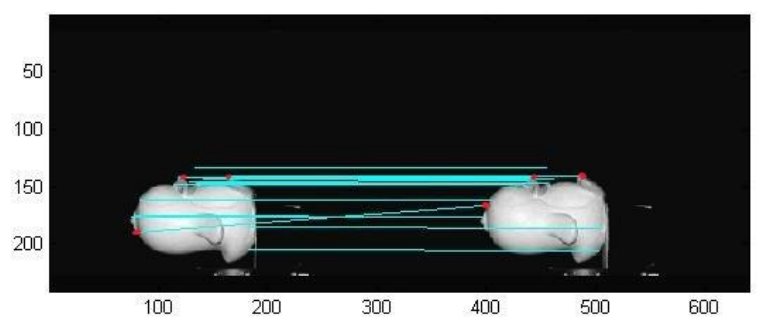

Figure 2. Matching Results of Image Toy (PCA-SIFT)

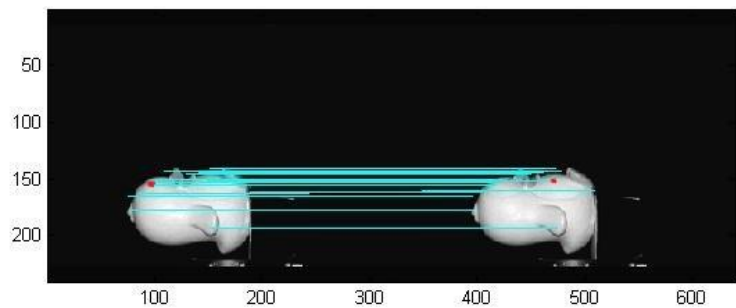

Figure 3. Matching Results of Image Toy (Improved 2DPCA-SIFT)

Table 1 gives the performance Comparison of PCA-SIFT and improved 2DPCA-SIFT for image toy. The number of key points found by PCA-SIFT is obviously less than improved that found by 2DPCA-SIFT algorithm. For image toy, PCA-SIFT found 161 key points, while improved 2DPCA-SIFT found 183 key points. Improved 2DPCA-SIFT got 24 correct pairs form 25 pairs key points, increasing the matching rate from $86.9 \%$ to 96\% In terms of matching rate, improved 2DPCA-SIFT algorithm is more better than PCA-SIFT. At the same time, our proposed matching algorithm saves computation time.

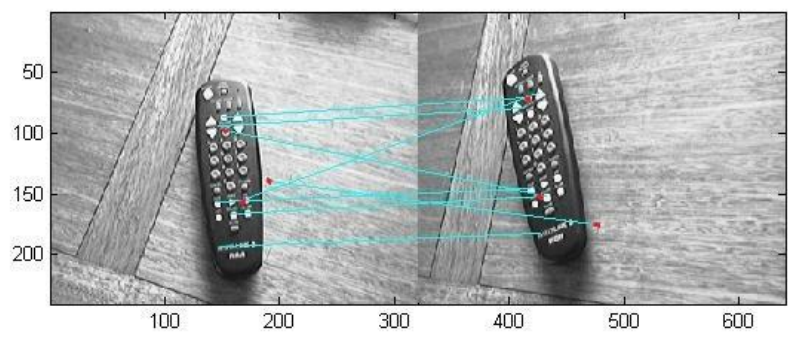

Figure 4. Matching Results of Image Telecontroller (PCA-SIFT)

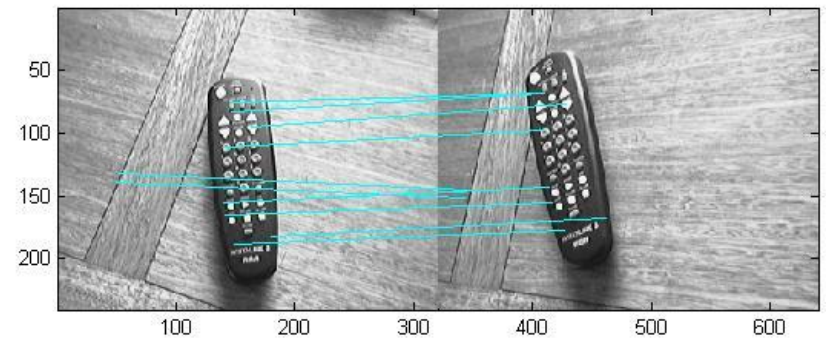

Figure 5. Matching Results of Image Telecontroller (Improved 2DPCA-SIFT) 
Table 1. Comparison of PCA-SIFT and improved 2DPCA-SIFT

\begin{tabular}{|c|c|c|c|c|c|}
\hline image & Algorithm & key points & $\begin{array}{c}\text { Matched } \\
\text { pairs }\end{array}$ & $\begin{array}{c}\text { Accuracy } \\
\text { rate }\end{array}$ & Time(s \\
\hline \multirow{2}{*}{ toy } & PCA-SIFT & 161 & $23(20)$ & $86.9 \%$ & 0.246 \\
\cline { 2 - 6 } & $\begin{array}{c}\text { Improved } \\
\text { 2DPCA-SIFT }\end{array}$ & 183 & $25(24)$ & $96 \%$ & 0.198 \\
\hline \multirow{2}{*}{$\begin{array}{c}\text { Tele } \\
\text { controller }\end{array}$} & $\begin{array}{c}\text { PCA-SIFT } \\
\text { Improved }\end{array}$ & 194 & $8(5)$ & $62.5 \%$ & 0.308 \\
\cline { 2 - 6 } & 216 & $10(10)$ & $100 \%$ & 0.264 \\
\hline
\end{tabular}

\section{Summary}

Improved 2DPCA-SIFT algorithm proposed in this paper presented low computational complexity. For local brightness, blurring, as well as rotation and scale transformations, our matching algorithm gave better results with strong robustness compared to PCA-SIFT algorithm, obviously reducing the running time and improving the matching accuracy.

\section{References}

[1] F. Zhou, "Scale-invariant feature (SIFT) transform-based image registration techniques", Kunming, Kunming University, (2010), pp. 1-3.

[2] D. G. Lowe, "Distinctive Image Features from Scale-Invariant Key points", International Journal of Computer Vision, vol. 60, no. 2, (2004), pp. 91-110.

[3] M. H. Rahman, M. R. Pickering, M. R. Frater and D. Kerr, "Texture feature extraction method for scale and rotation invariant image retrieval", Electronics Letters, vol. 48, no. 11, (2012), pp. 626-627.

[4] H. Bay, A. Ess, T. Tuytelaars and L. V. Gool, "Surf, Speeded Up Robust Features", Computer Vision and Image Understanding, vol. 110, no. 3, (2008), pp. 346-359.

[5] M. Lourenc, J. P. A. Barreto, F. Vasconcelos, "sRD-SIFT, key-point detection and matching in images with radial distortion", IEEE Transactions on Robotics, vol. 28, no. 3, (2012), pp. 752-760.

[6] G. Coppini and S. Diciotti, "Matching of medical images by self - organizing neural networks", Pattern Recognition Letters, vol. 25, no. 3, (2004), pp. 341-352 .

[7] A. Wang, N. Jiang and Y. Feng, "Face Recognition Based on Wavelet Transform and Improved 2DPCA", Higher Education Key Lab for Measuring \& Control Technology and Instrumentations of Heilongjiang, Harbin University of Science and Technology, (2013), pp. 2-3.

[8] J. Flusser and T. Suk, "A moment - based approach to registration of images with affinegeometric distortion”, IEEE Trans.Geo - Science and Remote Sensing, vol. 32, no. 2, (1994), pp. 382-387 .

[9] L. Juan, O. Gwun, "A comparison of SIFT, PCA-SIFT and SURF", International Journal of Image Processing, vol. 3, no. 4, (2009), pp. 143-152.

[10] J. Yang, D. Q. Zhang, A. F. Frangi and J. Y. Yang, "Two-dimensional PCA, a new approach to appearance-based face representation and recognition", IEEE Transactions on Pattern Analysis and Machine Intelligence, vol. 26, no. 1, (2004), pp. 131-137. 